\title{
Stress and mental health in neonatal intensive care units
}

\author{
R Kim Oates, Peter Oates
}

\begin{abstract}
The views of 34 neonatologists (a $78 \%$ response rate) and 192 neonatal intensive care nurses (a $66 \%$ response rate) were obtained on work, stress, and relationships in neonatal intensive care units. The survey was conducted by post and included Goldberg's General Health Questionnaire (GHQ). A comparison of the responses of neonatologists and nurses to 21 identical statements showed significant differences in 12. Most neonatologists felt that they involved nurses in critical patient care decisions, provided adequate pain relief for their patients, gave nurses adequate information on patients' progress after discharge, and were aware of little doctor-nurse conflict. However, the nurses' responses differed significantly in these areas, suggesting that the neonatologists may have a more rosy view of life in the neonatal intensive care unit than their nurse colleagues. Twenty seven per cent of neonatologists and $32 \%$ of nurses had GHQ scores indicating psychological dysfunction. The neonatologists who had dysfunctional scores differed from their colleagues in only one area surveyed - a higher proportion experienced conflict between the demands of their work and their personal lives.

(Arch Dis Child 1995; 72: F107-F110)
\end{abstract}

Keywords: mental health, neonatal intensive care units, neonatologists, nurses.

Work in intensive care units can be stressful. ${ }^{12}$ The stresses may be especially severe in neonatal intensive care units where there can be ethical as well as practical challenges, ${ }^{3}$ a high degree of burnout, ${ }^{4}$ competition between the demands of professional and personal life, ${ }^{5}$ and an excessive workload, contributing to stress and diminished job satisfaction. ${ }^{6}$

Working in a neonatal intensive care unit is a team effort, particularly between the neonatologists and the nursing staff. More has been written about the stresses experienced by nurses in these situations, ${ }^{7-9}$ but only one study has looked at similarities and differences in the stresses experienced by medical and nursing staff in neonatal intensive care units. ${ }^{5}$ None has compared the mental health of these two professional groups using a standard measure of mental health.

This study compared the views of a group of neonatologists and neonatal intensive care nurses using a questionnaire which included a standardised measure of general health. 1011

\section{Methods}

Two surveys based on previously published studies on stress among neonatologists and neonatal intensive care unit nurses were developed. The survey sent to neonatologists included 29 questions under the headings: work environment; patient care; technology; education and relationships; sources of stress. The nurses' survey had 42 questions grouped under similar headings. Twenty one of the questions on the neonatologists' survey were identical to those in the nurses' survey. The survey was piloted to ensure that questions were unambiguous and some modifications were made as a result of this pilot. For 23 of the statements in the survey the respondents were asked to circle one of four responses on a Likert scale of: strongly agree; agree; disagree; strongly disagree.

Each recipient was also asked to complete the General Health Questionnaire (GHQ40). ${ }^{1112}$ The GHQ is an efficient, reliable, and valid index of psychological impairment that has been validated in an Australian population where it achieved $87 \%$ sensitivity and $94 \%$ specificity with a misclassification rate of $7 \cdot 5 \%$. ${ }^{13}$ A large Australian community survey found that $13.5 \%$ of men and $18.7 \%$ of women had scores indicative of psychological impairment. ${ }^{14}$

Nurses were eligible for inclusion in this study if they worked in any of the six major neonatal intensive care units in the greater Sydney metropolitan area. This group comprised 291 nurses. Survey forms were sent to all paediatricians identified from the membership list of the Australian College of Paediatrics as being neonatologists or as having a major specialty interest in neonatology $(n=4)$. The surveys were completed anonymously.

For data analysis, the survey categories 'strongly agree' and 'agree' were combined as were the categories 'disagree' and 'strongly disagree' to give two broad categories, 'agree' and 'disagree' for each statement. Comparisons were made using the $\chi^{2}$ test or Fisher's exact test when cells contained small numbers. The GHQ was scored in accordance with the manual ${ }^{11}$ using a score of 5 or greater to indicate psychological impairment.

As the paediatricians came from all over Australia and the nurses came from neonatal units in one state, New South Wales, the responses of the New South Wales neonatologists were compared with those of the 
Table 1 Neonatologists in agreement with statements in the survey and comparison of those with normal (0-4) and high (5 or more) GHQ scorest

Neonatologist in agreement with statement

\begin{tabular}{|c|c|c|c|c|}
\hline \multirow[b]{2}{*}{ Statement } & \multicolumn{2}{|c|}{ Total } & \multirow{2}{*}{$\begin{array}{l}G H Q 0-4 \\
N o=\end{array}$} & \multirow{2}{*}{$\begin{array}{l}G H Q 5 \text { or more } \\
N o=\end{array}$} \\
\hline & No & $(\%)$ & & \\
\hline My ward based work area is well laid out & 22 & (65) & 16 & 6 \\
\hline My ward work area is overcrowded & 14 & (41) & 11 & 3 \\
\hline I involve nursing staff in patient care decisions that are non-critical & 30 & (88) & 22 & 8 \\
\hline There should be more nursing staff involvement in non-critical care decisions & 24 & (73) & 17 & 7 \\
\hline I involve nursing staff in patient care decisions that are critical & 33 & $(100)$ & 24 & 9 \\
\hline Nursing staff should be involved in these critical decisions & 34 & (100) & 25 & 9 \\
\hline The priority given to pain relief in my unit is adequate & 28 & $(80)$ & 19 & 9 \\
\hline Nurses have considerable influence in decisions to provide pain relief & 27 & (77) & 20 & 7 \\
\hline Considerable patient post discharge information is provided to the nurses & 20 & (59) & 16 & 4 \\
\hline Most of this post discharge information is provided by me & 16 & (47) & 12 & 4 \\
\hline I am completely up to date with my specialty & 21 & $(60)$ & 16 & 5 \\
\hline Keeping up to date is stressful & 19 & (54) & 11 & 8 \\
\hline Conflict between my job demands and needs of my personal life is a problem & 25 & (71) & $16^{\star}$ & $9 *$ \\
\hline I am involved in many non-work related activities & 19 & (54) & 16 & 3 \\
\hline I worry about my work when off duty & 19 & (54) & 12 & 7 \\
\hline Nursing understaffing is a problem in my unit & 21 & (62) & 14 & 7 \\
\hline Nurse understaffing is a source of stress in my work & 19 & (54) & 12 & 7 \\
\hline Nursing administration is supportive of the work in my unit & 25 & (74) & 16 & 9 \\
\hline The atmosphere in my unit is supportive & 32 & (94) & 23 & 9 \\
\hline Neonatologist/nurse conflict is a problem & 3 & (9) & 2 & 1 \\
\hline Junior doctor/nurse conflict is a problem in my unit & 13 & (39) & 9 & 5 \\
\hline Most conflict in my unit is due to communication problems between doctors and nurses & 12 & (34) & 7 & 5 \\
\hline My job is very satisfying & 33 & (97) & 25 & 8 \\
\hline
\end{tabular}

My job is very satisfying

${ }^{\star} \mathrm{p}<0 \cdot 02$. $\nmid$ Percentages differ slightly for similar numbers as the number of valid responses to statements varied.

neonatologists from other states. Where there was no significant difference between these two groups, their responses were combined and compared with the responses of the neonatal intensive care nurses.

\section{Results}

The response rate for neonatologists was $78 \%$ (35 usable responses from 45 neonatologists) and for nurses was $66 \%$ (192 usable responses from 291 nurses).

Table 1 shows the responses of the neonatologists to the 23 statements on the Likert scale. It also compares the results of those with a GHQ score of less than 5 with those whose scores of 5 or more suggest possible psychological dysfunction. The neonatologists believe they give high priority to pain relief $(80 \%)$ and to involving nursing staff in clinical decisions $(100 \%$ for critical decisions and $88 \%$ for non-critical decisions). Sixty per cent felt they were completely up to date in their specialty. Ninety seven per cent found their job satisfying, even though more than half worried about their work when off duty, and most (94\%) thought that the atmosphere in their unit was supportive. The neonatologists whose GHQ scores were higher than normal were significantly different from their colleagues in only one area - an increased amount of conflict between the demands of their job and their interpersonal life.

The last item in the survey also listed eight possible sources of stress and asked respondents to rank these in order of importance in their work. The eight sources of stress were: stress related to parental demands; insecurity about one's competence and knowledge; the health problems of the neonates; problems in the physical working environment; doctornurse relationships; administrative problems; peer relations; and understaffing or overwork. Understaffing and overwork was ranked as the major cause of stress, followed by administrative problems. The two lowest ranking items were peer relations and insecurity about competence and knowledge. There were no

Table 2 Neonatologists and nurses in agreement with statements

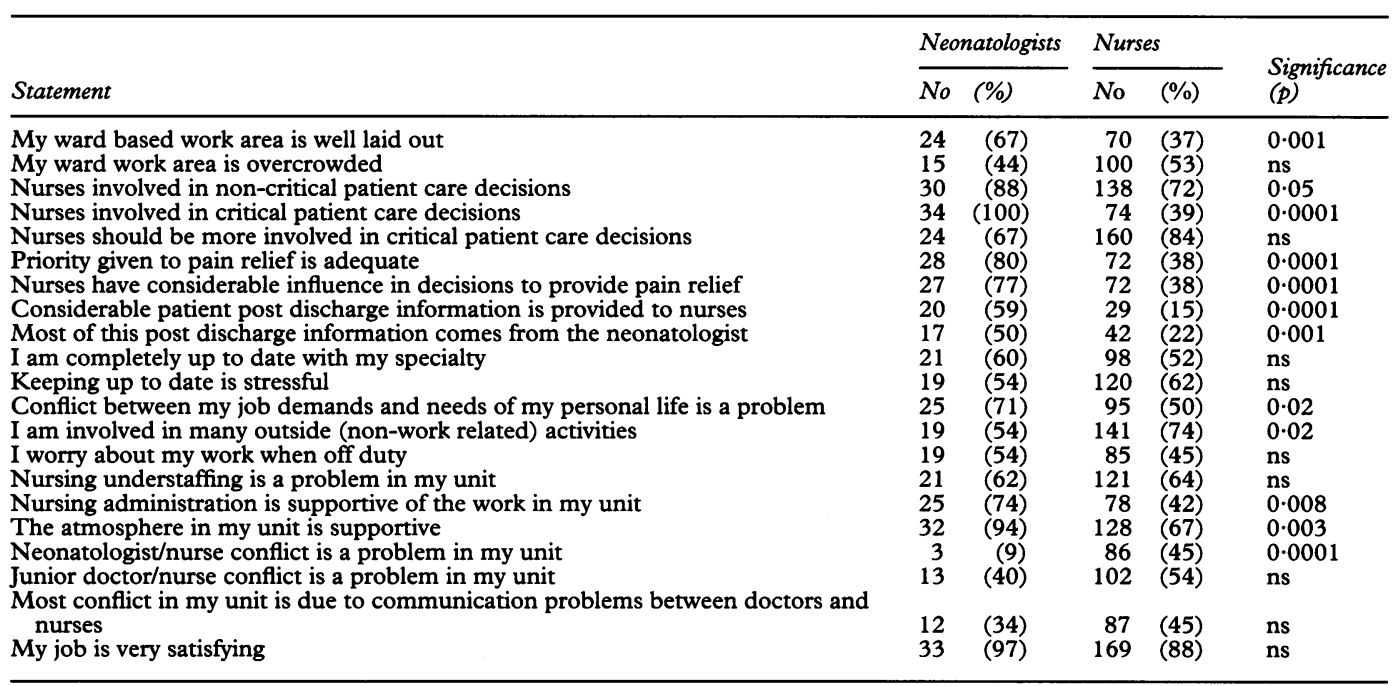


Table 3 Differences between responses by nurses with normal and high GHQ scores

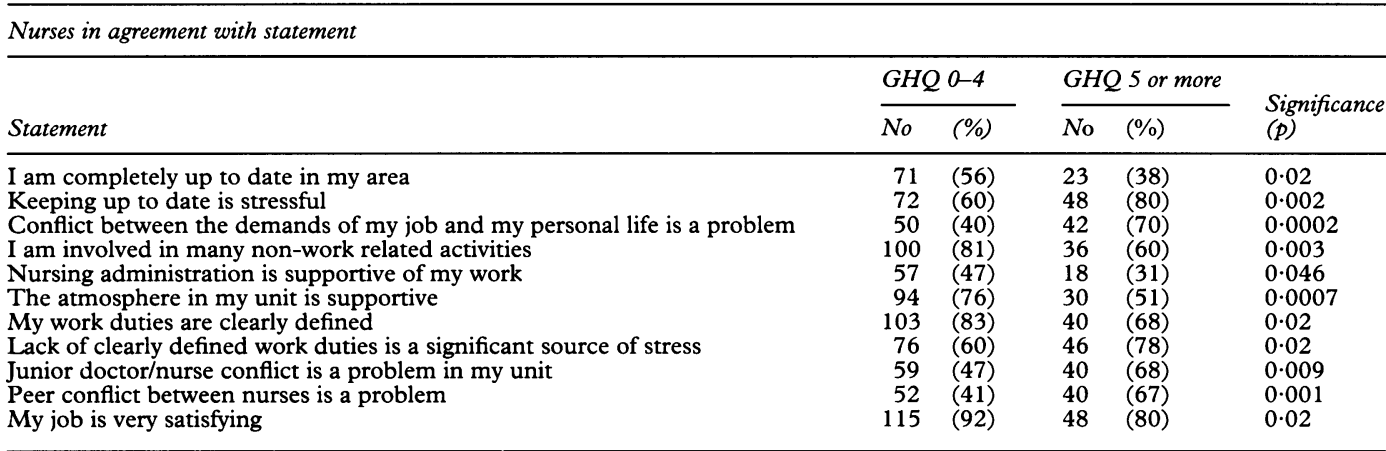

significant differences in these rankings between neonatologists who had high and low GHQ scores.

Of the 34 General Health Questionnaires that could be scored, nine $(26.5 \%)$ had a score of 5 or more. Ninety one per cent of the respondents were male. This result is significantly greater than the $13.5 \%$ of the Australian male population who have scores of 5 or more $(\mathrm{p}<0.04)$.

Twenty one statements in the neonatal intensive care nurses' survey were identical to those in the neonatologists' survey. As no significant differences were found between the responses of the New South Wales neonatologists and neonatologists from other states for these 21 questions, the responses of the total group of neonatologists were compared with the nurses' responses (table 2). In 12 of the 21 areas neonatologists and neonatal nurses had significantly different perceptions of work and relationships in the neonatal intensive care unit. For example, $67 \%$ of neonatologists felt that the ward work area was well laid out compared with $37 \%$ of nurses. All neonatologists believed they involved nurses in critical patient care decisions, compared with $39 \%$ of nurses. There was less difference in the perception of involvement in non-critical patient care decisions, with $72 \%$ of nurses feeling they were involved, compared with $88 \%$ of neonatologists believing they involved nurses in these decisions.

Neonatologists $(80 \%)$ were much more likely than nurses $(38 \%)$ to believe that adequate priority was given to pain relief. Only $38 \%$ of nurses felt that they were influential in decisions to provide pain relief compared with $77 \%$ neonatologists who felt nurses did influence their decisions in this area. Fifty nine per cent of neonatologists felt that the nurses received considerable information about their patients after discharge, with $50 \%$ of neonatologists believing they personally provided this information. This compares with only $15 \%$ of nurses believing they received an adequate amount of such information, with $22 \%$ believing that most of this information came from the neonatologists.

The differences in perception about their personal lives, although still significant, were less noticeable. Seventy one per cent of neonatologists and $50 \%$ of nurses felt that conflict between their job demands and the needs of their personal lives were a problem, while more nurses $(74 \%)$ than neonatologists (54\%) felt that they were involved in many non-work related activities.

Less than half $(42 \%)$ the nurses felt that the nursing administration was supportive of the neonatal intensive care unit compared with almost three quarters $(74 \%)$ of neonatologists with this view. The atmosphere in the unit was felt to be supportive by $94 \%$ of neonatologists compared with $67 \%$ of nurses, while many more nurses $(45 \%)$ than neonatologists $(9 \%)$ felt that conflict between neonatologists and nurses was a problem.

When the nurses were asked to rank the same eight potential problems as the neonatologists, the highest ranking problem was identified as understaffing and overwork, followed by concern about the wellbeing of their patients. The lowest two concerns from the list were the same as for the neonatologists - insecurity about their knowledge and competence, and peer relationships.

Fifty nine $(32 \%)$ of 186 valid nurses' responses on the GHQ had scores of 5 of more. This result is not significantly different from the $26 \cdot 5 \%$ found for the neonatologists, but it is considerably higher than the $19 \%$ that would be expected from a random sample of the Australian population $(\mathrm{p}<0.0001)$.

When the responses of the nurses with high GHQ scores were compared with those who had normal GHQ scores, there were significant differences on 12 items (table 3 ). The nurses with the higher GHQ scores felt it more stressful to be involved in critical patient care decisions, felt less up to date, and experienced more stress in trying to keep up to date. They were less involved in non-work related activities and more likely to experience conflict between the demands of the job and their personal lives. They were more likely to feel their work duties were not clearly defined and to feel that this was a source of stress. A lack of support within the unit and by nursing administration was more likely to be felt by this group. They found their jobs less satisfying and were more likely to see conflict between resident medical staff and nurses as well as perceiving more peer conflict between the nurses.

\section{Discussion}

Some of the responses of the neonatologists in this survey are consistent with those of Clarke 
et $a l,{ }^{6}$ who found that $50 \%$ of neonatologists experienced stress at work, with an excessive workload being the major cause of this stress. However, one sixth of neonatologists in that survey were not satisfied with their career, in contrast to the findings of the present study where $97 \%$ of neonatologists regarded their job as being very satisfying. The neonatologists in this study ranked understaffing and overwork as well as administrative problems as their major sources of stress. These findings are consistent with those of Astbury and $\mathrm{Yu},{ }^{5}$ who found that neonatologists were more likely than nurses to experience stress from the competing demands of personal and professional life; nurses, who are generally in a subordinate position to the doctors, were more likely to experience difficulties working with doctors than vice versa.

Significantly more nurses than neonatologists felt that there were problems in their physical work environment as well as problems in terms of lack of support, factors which have been shown to be associated with job burnout in nurses. ${ }^{7}$ Lack of feedback from senior staff to nursing staff, a factor associated with nursing dissatisfaction, ${ }^{15}$ was a problem for $85 \%$ of nurses, even though over half $(59 \%)$ of the neonatologists believed they provided this. Discrepancies such as this suggest that the neonatologists have a somewhat more rosy picture of life in the neonatal intensive care unit than the nurses, who are nearly always at the 'front-line' for the duration of their working day.

The nurses felt they did not exercise as much authority and responsibility, especially in regard to being involved in critical care decisions and in administering pain relief, as the neonatologists believed they were giving them. While not many neonatologists felt that doctor-nurse relationships were a problem, about half the nurses felt that there were difficulties resulting from communications and conflicts between neonatologists and nurses. While there may well be errors of perception on both sides, these results suggest that the neonatologists' view of the level of conflict, communication, and responsibility may not be as favourable as they think.

Some of these findings are not surprising. What is perhaps more surprising is the higher than would be expected proportion of neonatologists and neonatal intensive care nurses who had GHQ scores indicative of psychological stress. It is clear that working in an intensive care situation, where daily decisions about life and death are made, must be stressful, but the GHQ scores indicate that these stresses, which are accepted as going with the job, affect the personal lives of many of the people working in these units. This, along with the evidence that staff who work in critical care areas are at increased risk of developing illness, ${ }^{16}$ are factors which are likely to add to the workload and associated stress for other staff, thus forming a vicious cycle.

It could be argued that there may be factors in neonatal intensive care work which attract people with higher GHQ scores, although there is no documented evidence for this, so that a cause and effect relationship between GHQ scores and work, stress, and relationships is not necessarily established from this study. The stresses are likely to be greater for nurses who have fewer opportunities than doctors to seek relief from the difficulties that go with working in this atmosphere. While doctors often have 'off-service' periods, opportunities to teach, carry out research and attend meetings outside the unit, nurses have fewer such opportunities. The availability of nursing support groups, ${ }^{817}$ provided these groups are supported by nursing administration and initiated by nurses rather than at the suggestion of doctors, ${ }^{18}$ and increased recognition by medical staff of the importance of consulting with and listening to their nursing colleagues may assist in ameliorating some of these stresses.

We thank Rogan McNeil for his expert statistical advice.

1 Frader JE. Difficulties in providing intensive care. Pediatrics 1979; 64: 10-6.

2 Walker CHM. Neonatal intensive care and stress. Arch Dis Child 1982; 57: 85-8.

3 Duff RS, Campbell AGM. Moral and ethical dilemmas in the special care nurser. $N$ Engl $\mathcal{F}$ Med 1973; 289: 890-4.

4 Marshall RE, Kasman C. Burnout in the neonatal intensive care unit. Pediatrics 1980; 65: 1161-5.

5 Astbury J, Yu VYH. Determinants of stress for staff in a neonatal intensive care unit. Arch Dis Child 1982; 57: 108-11.

6 Clarke TA, Maniscalco WM, Taylor-Brown S, Roghmann KJ, Shapiro DL, Hannon-Johnson C. Job satisfaction and stress among neonatologists. Pediatrics 1984; 74: 52-7.

7 Constable JF, Russell DW. The effect of social support and the work environment upon burnout among nurses. the work environment upo
f Hum Stress 1986; 12: $20-6$.

8 Oehler JM, Peter MA, Seyler S. Support groups: are they really helpful in dealing with NICU stress? Neonatal Network 1989; 8: 21-5.

9 Rosenthal SL, Schmid KD, Black MM. Stress and coping in a NICU. Research in Nursing and Health 1989; 12: 257-65.

10 Goldberg DP, Cooper B, Eastwood M, Kedward H, Shepherd M. A psychiatric interview suitable for use in community surveys. Br $\mathcal{F}$ Social Prevent Med 1970; 24: 18-26.

11 Goldberg D. Manual of the General Health Questionnaire. Windsor: NFER Publishing Company, 1978.

12 Huppert FA, Walters DE, Day NE, Elliott BJ. The factor structure of the General Health Questionnaire (GHQ-30) A reliability study on 6317 com

13 Tennant C. The General Health Questionnaire: A validation of psychological impairment in Australian population. Med F Aust 1977; 2: 392-4.

14 Finlay-Jones RA, Burvill PW. The prevalence of minor psychiatric morbidity in the community. Psychol Med 1977; 7: 474-89.

15 Nichols KA, Springford V, Searle J. An investigation of distress and discontent in various types of nursing. $\mathcal{F} A d v$ Nursing 1981; 6: 311-8.

16 Oskins $\mathrm{S}$. Identification of situational stressors and coping methods by intensive care nurses. Heart and Lung 1979; 8: methods

17 Weiner MF, Caldwell T. The process and impact of an ICU nurse support group. Int $\mathscr{f}$ Psychiatry Med 1983; 13: 47-55.

18 Weiner MF, Caldwell T, Tyson J. Stress and coping in ICU nursing: Why support groups fail. Gen Hosp Psychiatry 1983; 5: 179-83. 